\title{
Une approche économique des dégâts de gibier. Indemnisation, prix et propriété
}

An Economic Approach to Damage Caused by Big Game. Compensation, Price and Property

\section{Laurent Carnis et François Facchini}

\section{(2) OpenEdition}

\section{Journals}

Édition électronique

URL : http://journals.openedition.org/economierurale/3393

DOI : 10.4000/economierurale.3393

ISSN : 2105-2581

\section{Éditeur}

Société Française d'Économie Rurale (SFER)

Édition imprimée

Date de publication : 1 mars 2012

Pagination : 126-142

ISSN : 0013-0559

Référence électronique

Laurent Carnis et François Facchini, «Une approche économique des dégâts de gibier. Indemnisation, prix et propriété ", Économie rurale [En ligne], 327-328 I janvier-mars 2012, mis en ligne le 01 mars

2014, consulté le 19 avril 2019. URL : http://journals.openedition.org/economierurale/3393 ; DOI :

10.4000/economierurale.3393 


\section{Une approche économique des dégâts de gibier Indemnisation, prix et propriété}

Laurent CARNIS • Chargé de recherche, Institut français des sciences et technologies des transports de l'aménagement et des réseaux, département économie et sociologie des transports, IFSTTAR-DEST laurent.carnis@ifsttar.fr

François FACCHINI • Centre d'économie de la Sorbonne, Université Paris 1, axe institution facchini@univ-paris1.fr http://ces.univ-paris1.fr/membre/facchini

'objectif de cet article est d'expliquer L'évolution du montant des dépenses d'indemnisation payées par les chasseurs aux agriculteurs pour les dégâts causés par le grand gibier en France. Cette analyse exclut de son champ d'investigation ce qui est dénommé par chasse commerciale.

Généralement, l'évolution des indemnités versées par les chasseurs est expliquée sans référence à la théorie économique des prix et/ou à l'effet du droit sur les comportements des agents, autrement dit des agriculteurs et des chasseurs. La littérature, peu abondante, sur ce sujet (Baubet et al., 2008 ; Pfaff et Saint Andrieux, 2008 ; Le Goffe et Roppars, 2008 ; Baubet, Vassant, Brandt, et Maillard, 2008) explique, en effet, la croissance des indemnités versées par les chasseurs aux agriculteurs en rendant compte, d'une part, des raisons de l'augmentation du nombre des dégâts de gibier et, d'autre part, de l'importance du préjudice indemnisable. Elle insiste, à cette occasion, sur les facteurs qui favorisent la hausse du nombre de grands gibiers comme les ressources alimentaires et spatiales dont disposent ces derniers, l'évolution des pratiques cynégétiques et sur les facteurs qui déterminent le montant des préjudices : les salaires des estimateurs départementaux et des experts nationaux ainsi que le prix des biens agricoles.

Il paraît, cependant, raisonnable de supposer que le coût du prélèvement pour les chasseurs, d'une part, et l'évolution du cadre juridique, d'autre part, ont des effets sur l'évolution des dépenses d'indemnisation.

Une décision en univers de rareté présente toujours un coût d'opportunité. Ce choix sacrifie toujours une multitude d'opportunités pour en exploiter une seule. Cela conduit à s'interroger sur les informations dont dispose le chasseur, mais également sur leur qualité, lorsqu'il prend sa décision d'abattre un animal. Le prix de marché, généralement, informe les agents sur leur consentement à payer, autrement dit leur coût d'opportunité. Le prix de marché est, en ce sens, un médium de communication. Or ce qui caractérise l'activité de la chasse est l'absence d'un prix de marché pour chasser le grand gibier, absence qui s'explique par le statut de res nullius de ce dernier. En l'absence de droits de propriété bien définis, le chasseur et l'ensemble des parties au litige sont privés d'une information sur ce que les individus sont prêts à payer pour abattre ou protéger un grand gibier. Il convient donc d'évaluer aussi les conséquences de cette absence de prix de marché sur la dynamique des dépenses d'indemnisation.

L'évolution du cadre juridique a aussi vraisemblablement des effets sur les comportements des agents et in fine sur l'évolution du montant des indemnités. L'économie du droit et plus particulièrement des règles de responsabilité (Cooter et Ulen, 2004 ; Shavell, 1987) propose un cadre conceptuel utile pour rendre compte 
de ces effets. Depuis la loi de 1968, les dégâts de gibier causés aux cultures sont à la charge des fédérations de chasse ${ }^{1}$ (Charlez, 1994 ; Chassin, 2001 ; Delfour $1998)^{2}$. L'indemnisation par les fédérations départementales des chasseurs des dégâts causés par les sangliers et les grands gibiers est encadrée par la loi chasse du 6 juillet 2000, modifiée par la loi du 23 février 2005 et codifiée dans le Code de l'environnement par les articles L. 426-1 à L. 426-6.

L'indemnisation n'est possible que pour les seuls sangliers et grands gibiers à l'exception de tous les autres (art. L. 426-1). Par grand gibier, il faut comprendre les animaux soumis légalement au plan de chasse (cerf, cerf sika, chevreuil, daim, mouflon, chamois et isard). L'indemnisation n'est due qu' aux seuls exploitants agricoles (art. L. 426-1) et ne concerne que les récoltes ou les travaux de remise en état (art. L. 426-1). Ce qui exclut toute indemnisation des pertes indirectes (détérioration du matériel, dégâts aux silos, temps de personnels...). La loi française transfère donc aux chasseurs la responsabilité des dommages sans leur attribuer un droit de propriété sur celui-ci, même s'il existe des mécanismes leur octroyant des droits de prélèvement dans le cadre de l'activité de chasse.

Cette législation définit une forme de responsabilité collective stricte. Responsabilité collective stricte, dans la mesure

\footnotetext{
1. Les dommages occasionnés par le grand gibier lors de collisions routières sont indemnisés par les assurances. Les dommages occasionnés aux forêts sont quant à eux internalisés par leurs propriétaires. 2. L'indemnisation administrative est effectuée à partir d'un fonds géré par ONCFS (Office national de la chasse et de la faune sauvage) qui a remplacé l'ONC (Office national de la chasse) en 2000. Ce fonds est abondé par les redevances cynégétiques et les cotisations des fédérations de chasse. Pour financer les indemnisations, des taxes sont prélevées auprès des chasseurs (art. L. 426 - 5 alinéa 3 du Code de l'environnement).
}

où l'indemnisation s'établit sans égard à une faute commise, et laquelle est mutualisée par un collectif représenté par les chasseurs. Il est utile de s'interroger sur les conséquences de cette règle de responsabilité sociale des chasseurs sur l'évolution des indemnités qu'ils versent aux agriculteurs, car la théorie économique de la responsabilité montre que chaque règle de responsabilité à des effets incitatifs différents. L'absence de responsabilité des agriculteurs dans la plupart des cas ne les incite pas, par exemple, à engager des dépenses de précaution. Une règle qui renforcerait leur responsabilité les inciterait, au contraire, à faire plus attention. La dimension collective de la responsabilité doit aussi avoir des effets sur les stratégies des acteurs et des chasseurs en particulier. La socialisation du risque de dégât de gibier devrait, en effet, favoriser des stratégies de passager clandestin qui pourraient expliquer la croissance du montant des indemnités payées par les chasseurs.

Cet article se propose donc de présenter, dans une première partie, les explications traditionnelles de l'évolution du montant des dépenses d'indemnisation payées par les chasseurs aux agriculteurs pour les dégâts causés par le grand gibier en France. Les deuxième et troisième parties se proposent de développer des explications qui donnent une place au raisonnement économique à ces catégories. La croissance des dépenses d'indemnisation n'aurait plus alors seulement pour cause l'évolution du prix des biens agricoles, celle du salaire horaire, les ressources alimentaires et spatiales à disposition des animaux, les pratiques cynégétiques, le tableau de chasse, le nombre des chasseurs, le prix du permis de chasse, mais aussi le coût des prélèvements et la mise en œuvre d'une règle de responsabilité sociale des chasseurs. Autant de variables qui interagissent dans un cadre institutionnel spécifique. 
Figure 1. Évolution comparée du montant total des indemnisations versées pour les dommages causés par le grand gibier et des prélèvements de sangliers en France depuis 1970

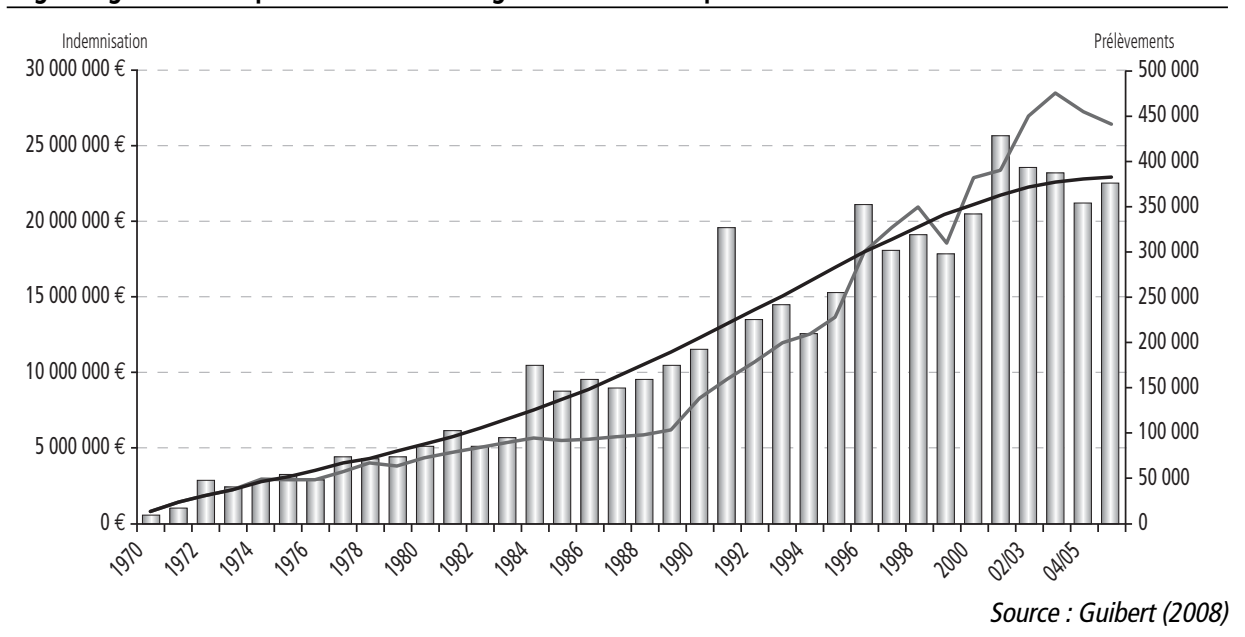

\section{Les explications a-économiques de la dynamique des indemnités}

L'évolution de la totalité des indemnités versées aux agriculteurs (toutes espèces confondues) pour la France augmente jusqu'en 2000 pour stagner depuis (figure 1). "La part du sanglier dans ces dégâts représente environ $83 \%$ du montant total de l'indemnisation soit près de 19 millions d'euros après vient le cerf pour $14 \%$ et le chevreuil pour environ $3 \%$. Les dégâts concernent principalement les maïs (35\%), les céréales (25\%) et les prairies (24\%) 》 (Guibert, 2008, p. 74). Il existe, par ailleurs, de très fortes disparités départementales et communales. Trois cent soixante-sept communes (soit $1 \%$ des communes françaises) concentrent le quart de la totalité des dégâts de sangliers (Ibid.).

La nature des dégâts dépend du type de végétation (forêt ou espaces cultivés) et du type d'animal (sanglier, cerf ou chevreuil).

- En forêt, les dégâts relèvent essentiellement des cervidés (cerfs, biches, chevreuils), car les sangliers qui fouissent le sol ne provoquent pas de dégâts sur la végétation sauf dans des situations exceptionnelles de trop grande densité. L'impact des cervidés sur l'économie forestière reste toutefois assez mal connu, parce qu'il n'existe aucune indemnisation des pertes subies par les propriétaires. Ce point ne sera pas développé, car ne faisant pas l'objet du présent article.

- L'impact du grand gibier sur l'économie agricole est mieux connu en raison des règles d'indemnisation des dommages subis par les agriculteurs. Les dégâts sont à la fois le fait des cervidés et des sangliers. Les principales causes de dégâts sont le piétinement des cultures et les sangliers qui fouissent le sol. Les dégâts de sangliers aux cultures agricoles sont essentiellement d'ordre alimentaire. Ils sont réalisés lors de la recherche de la prise de nourriture (Vassant et Brandt, 2008).

Les dégâts de gibier peuvent être indemnisés, si l'agriculteur déclare le dommage et dépose un dossier d'indemnisation tout en respectant la procédure. Un expert est mandaté pour déterminer l'importance du dommage, qui donnera éventuellement lieu à une indemnisation pour des pertes subies. D'un point de vue formel, le montant des indemnisations versées par les chasseurs dépend du montant du 
préjudice indemnisable estimé pour chaque dossier et du nombre des dossiers déposés.

Le montant du préjudice indemnisable dépend positivement du prix des biens agricoles endommagés (relation positive), des conditions de rémunération des estimateurs départementaux et des experts nationaux qui constatent les dégâts (art. R. 426-19), et du prix de l'heure de travail pour remettre en état les terres endommagées. Ce sera l'objet de la première section.

Le nombre des dossiers est fonction du nombre de grands gibiers. Le nombre des grands gibiers dépend positivement des ressources dont dispose le grand gibier : les ressources naturelles et le garde mangé qui constitue la production agricole, ce que décrit la section II et négativement des pratiques cynégétiques, autrement dit du nombre des prélèvements, objet de la section III.

\section{Montant des préjudices et évolution des dépenses d'indemnisation}

\section{Le coût de la procédure}

Le montant des indemnisations versées par les chasseurs dépend des règles d'indemnisation. L'indemnisation n'est possible que pour les dégâts causés par les sangliers et les grands gibiers (art. L. 426-1) et ne concerne que les récoltes. Elle n'est pas envisageable lorsque les animaux viennent du propre fonds du réclamant (art. L. 426-2). Un seuil de déclenchement de 76 euros est appliqué pour toute procédure d'indemnisation (art. R. 426-11, Code de l'environnement). Les fédérations départementales des chasseurs déterminent et proposent à la victime un montant d'indemnité après qu'une évaluation du dommage ait été réalisée par un expert indépendant et dûment mandaté (art. L. 426-5). L'indemnité est déterminée en fonction des barèmes départementaux des récoltes endommagées, qui sont fixés par la Commission départementale de la chasse et de la faune sauvage. Cette commission est tenue, cependant, de respecter les fourchettes de barèmes d'indemnisation des denrées et des travaux fournies par la Commission nationale d'indemnisation des dégâts de gibier. Par ailleurs, une franchise de $5 \%$ est retenue sur le préjudice indemnisable estimé. Les fourchettes proposées par la Commission nationale tiennent compte de l'évolution du prix des denrées. Les dépenses d'indemnisation des chasseurs sont, pour cette raison, d'autant, plus importantes que le prix du maïs et des autres cultures sont élevés. Les pertes directes dépendent donc du prix de la production détruite.

\section{Les prix des biens agricoles}

De 1991 à 2004, les prix payés aux producteurs ont baissé de $4 \%$ à $6 \%$ par an en euros constants pour les céréales (blé tendre et maïs), comme pour les oléagineux (colza et tournesol) (Desbois et Legris, 2007). Les marges nettes par hectare du blé tendre et du maïs suivent des évolutions proches. Jusqu'en 1996, le blé tendre apparaissait plus rémunérateur que le maïs. C'est l'inverse qui prévalait dans les années 2000, le maïs prenant l'avantage (Ibid.) Ceci signifie que la valeur d'une même récolte diminue compte tenu de l'évolution du prix des céréales et des oléagineux, et donc que les pertes subies pour un même dégât sont plus faibles.

En conséquence, on peut avancer l'hypothèse raisonnable que ce n'est pas tant la hausse de la valeur moyenne du dommage qui est l'élément déterminant dans l'augmentation constatée des indemnisations versées pour dégâts de gibier que l'accroissement du nombre de dossiers à indemniser, et donc des variables déterminant ultimement le dépôt de dossiers de demande d'indemnisation. Ainsi, la hausse du montant des dépenses d'indemnisation s'expliquerait plutôt par un effet quantité (nombre de préjudices indemnisables) que par un effet «prix ». Cela signifie également qu'un renchérissement des prix des céréales conduirait à 
un niveau d'indemnisation encore plus important en termes financiers.

\section{Ressources alimentaires et spatiales disponibles}

L'effet quantité ou l'accroissement du dépôt du nombre de dossiers d'indemnisation s'explique principalement par le nombre de grands gibiers et donc par sa prolifération. En effet, la probabilité d'avoir un dégât de gibier est d'autant plus forte que le nombre de grands gibiers est élevé ceteris paribus. La demande de réparations (le nombre des dossiers déposés par les plaignants) doit alors augmenter avec le nombre des sangliers.

\section{Le nombre de grands gibiers}

Il dépend de leurs ressources alimentaires et spatiales, des ressources hors biens agricoles dont dispose l'animal. Si les fruits de la forêt ne peuvent pas nourrir la population de sangliers, celle-ci sera d'autant plus encline à aller se nourrir dans les espaces cultivés. Le développement d'une espèce animale dépend des ressources dont elle dispose (Baubet et al., 2008,). En ce qui concerne le sanglier, il constitue une espèce sédentaire et omnivore. La taille des domaines vitaux s'établit d'après leurs études entre 500 et 4000 hectares (Baubet et al., op. cit.). Il se nourrit à $95 \%$ de matière végétale (de fruits tels les glands, les faines et les châtaignes, de céréales et de maïs). En conséquence, le nombre de grands gibiers résulte de la cueillette et de la proximité des forêts (espace vital) au garde mangé agricole.

L'évolution de la répartition physique du territoire en France de 1992 à 2004 indique que les sols boisés ont augmenté de 3,3\%, les sols agricoles ont diminué de $2,9 \%$ et les sols bâtis de 17,6\%. La croissance des sols bâtis traduit l'artificialisation de l'espace français $(+17,6 \%$ sur la période), laquelle n'est pas favorable à la dynamique démographique des grands gibiers. La baisse des surfaces agricoles utiles $(-2,9 \%)$ et la hausse des sols boisés $(3,3 \%)$ sont des évolutions indifférentes pour les grands gibiers qui peuvent se nourrir en forêt ou sur les espaces cultivés. La hausse plus importante des sols boisés devrait cependant être favorable à la baisse des dégâts de gibier indemnisés, car elle donne aux grands gibiers un espace vital plus important. Ces conclusions ne sont pas cependant totalement convaincantes, car elles ne tiennent pas compte des évolutions par zone de la répartition physique du territoire. Des approches plus locales sont, pour cette raison, utiles.

\section{L'évolution des dépenses d'indemnisation}

L'analyse empirique des dégâts agricoles réalisée au niveau départemental par Le Goffe et Ropars (2008) tient compte en partie de ces évolutions contrastées. Elle cherche en effet à expliquer l'évolution des dégâts de sanglier sur la période 19912003, en testant au niveau départemental une fonction de dommage pour chaque type de culture (équation 1).

$$
\begin{aligned}
& D C_{i}=a_{0}+a_{1} P S \times S C_{i}+a_{2} P S \times S F \\
& +a_{3} \frac{P S S F}{S A U}+\sum_{k} b_{k} P S R_{k}
\end{aligned}
$$

avec $\left(D c_{i}\right)$ représentant les dommages pour la culture $(i),(P S)$ les prises annuelles de sanglier pour le département, $\left(S C_{i}\right)$ la surface départementale de la culture $(i)$ endommagée, $(S F)$ la surface de forêt, $(S A U)$ la surface agricole utile du département et $\left(R_{k}\right)$ un indicateur régional.

Leur fonction de dommages est estimée pour les céréales, le maïs, les prairies, les oléagineux et la vigne. Les résultats obtenus pour les oléagineux et la vigne s'avèrent très peu significatifs. « Le modèle linéaire estimé explique $56 \%$ de la variation des dommages agricoles sur le maïs (grain et fourrage), près de $70 \%$ de la variation des dommages sur les autres céréales, mais seulement $35 \%$ de la varia- 


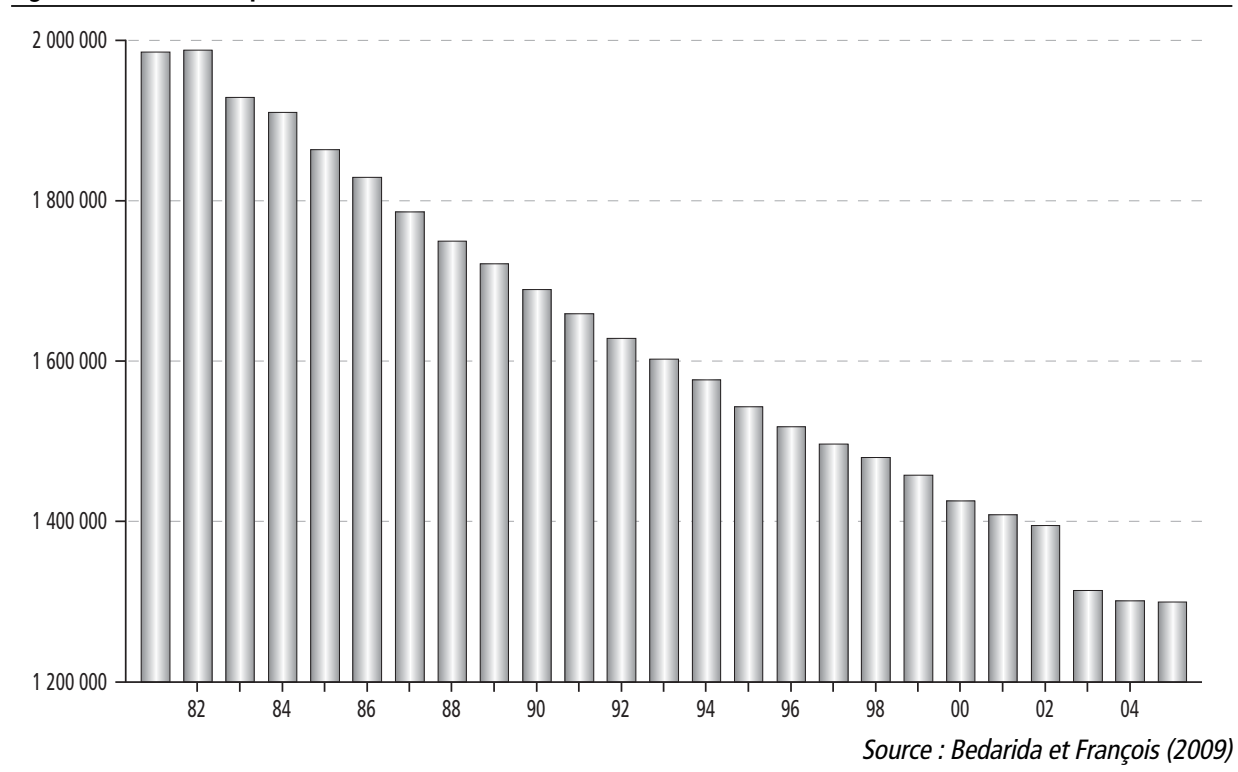

tion des dommages sur les prairies » (Ibid.). Les variables explicatives les plus significatives statistiquement pour comprendre l'importance des dommages causés aux cultures sont les surfaces de cultures et de forêt. Une surface cultivée plus importante conduit à un accroissement des dommages causés par le grand gibier. Une surface boisée plus conséquente est associée à une réduction des dommages causés. Les résultats obtenus au niveau départemental corroborent l'hypothèse établie pour le niveau national. Ils se révèlent fort intéressants puisqu'ils permettent d'isoler l'effet associé à l'évolution des ressources alimentaires et spatiales sur les dommages.

En conséquence, les départements où les surfaces boisées sont plus importantes, ceteris paribus, sont ceux pour lesquels le montant des dommages sera le plus faible. En revanche, les départements où les surfaces cultivées sont les plus importantes, ceteris paribus, sont ceux où le montant des dommages sera le plus élevé. En conséquence, le montant des dommages n'est pas indifférent aux choix et aux stratégies productives des agriculteurs, ce qu'avait d'ailleurs reconnu un rapport du Conseil économique et social de $2002^{3}$.

\section{Pratique cynégétique}

\section{Le nombre de grands gibiers}

Il dépend aussi du niveau des prélèvements effectués par les chasseurs. Les prélèvements sont d'autant plus importants que le nombre des prédateurs de grands gibiers est élevé. Le seul prédateur de grands gibiers dans la plupart des départements français, hors départements de montagne, est l'homme. Or le nombre de chasseurs baisse depuis l'année 1975 qui constitue l'année la plus élevée en termes d'effectifs. Le nombre de chasseurs s'établissait en effet à environ 2,2 millions en 1975. À présent, il y a moins de 1,3 million de chasseurs, soit une diminution conséquente de $40 \%$. Concernant le nombre de permis validés, il a chuté de $35 \%$ entre 1981 et 2005 (figure 2) (Bedarida et François, 2008).

3. Avis adopté par le Conseil économique et social au cours de sa séance du mercredi 11 décembre 2002, p. II. 130 et s. 


\section{Figure 3. Tableau national de chasse pour le cerf}

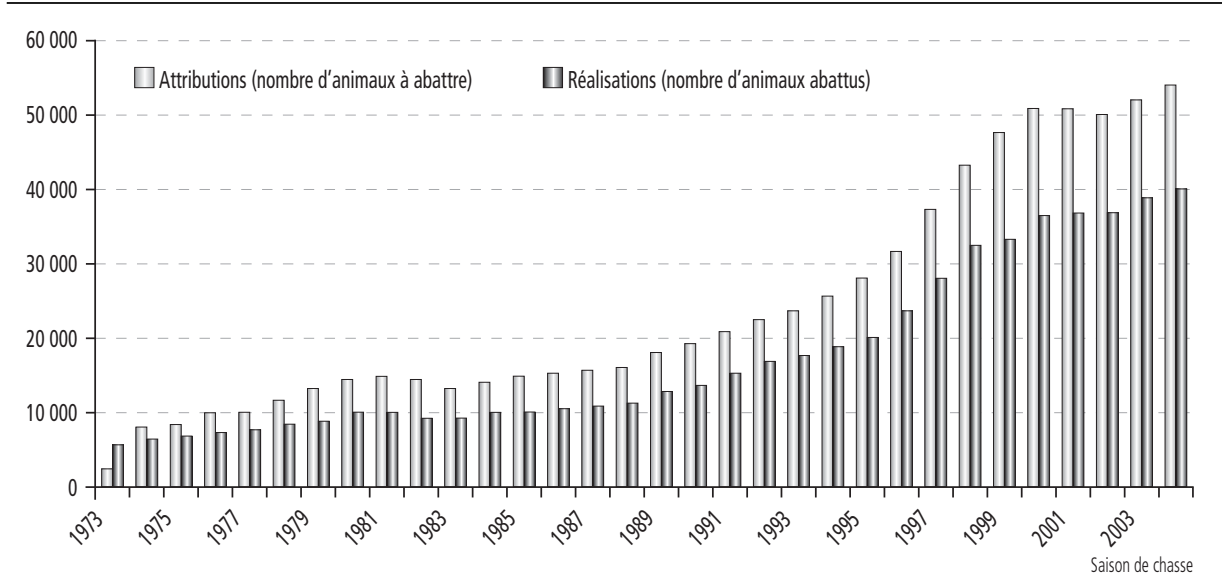

Source : Observation et statistiques de l'environnement ${ }^{5}$

\section{Figure 4. Tableau national de chasse pour le chevreuil}

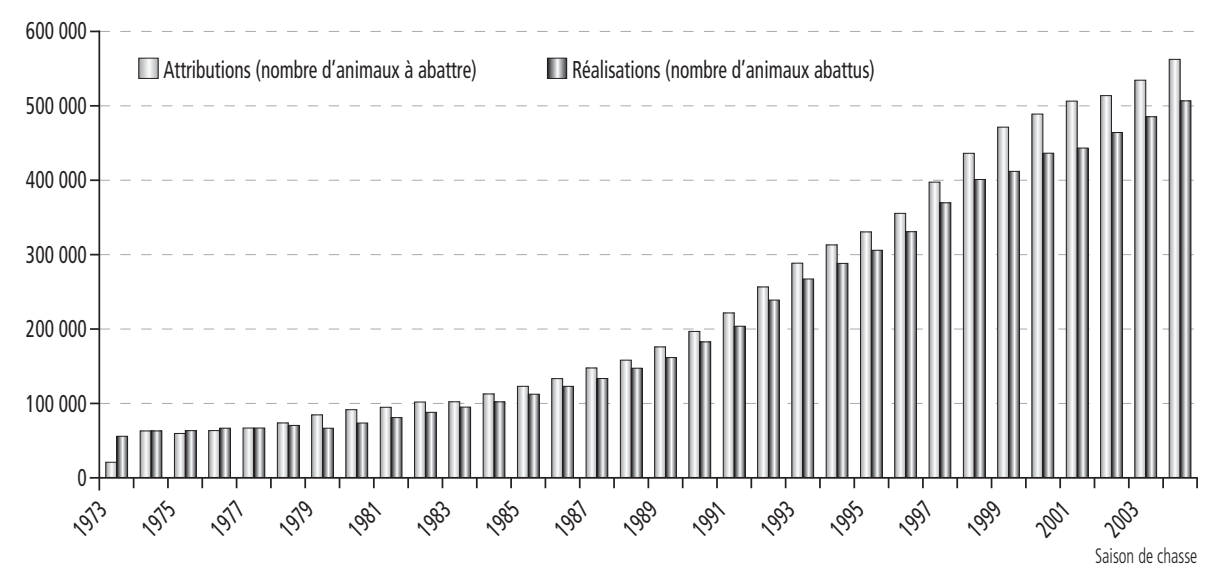

Quant aux nombres des prélèvements, ils augmentent pour le chevreuil, le cerf et le sanglier sur la période 1973-2005.

1. Le nombre de cerfs abattus a été multiplié par 3,6 entre 1985 et 2000 (figure 3$)^{4}$. Les prélèvements restent cependant globalement inférieurs à l'accroissement naturel des populations qui continuent de se développer. La popu-

4. Chiffre donné par l'Observatoire statistique de l'environnement. Site consulté le $1^{\text {er }}$ février 2009 , http://www.ifen.fr/donnees-essentielles/nature-etbiodiversite/faune-et-flore/les-cervides.html?taille=.
Source : Observation et statistiques de l'environnement ${ }^{6}$

lation de cerfs est, de plus, très inégalement répartie sur le territoire national, puisque le quart des effectifs est concentré sur $9 \%$ de la superficie totale occupée et la moitié sur $26 \%$ des surfaces occupées.

2. Sur la même période, le nombre des chevreuils abattus a été multiplié par 4 (figure 4). Les populations de ces deux espèces et le niveau des prélèvements

5. Consultation du site en février 2009.

6. Consultation du site en février 2009. 


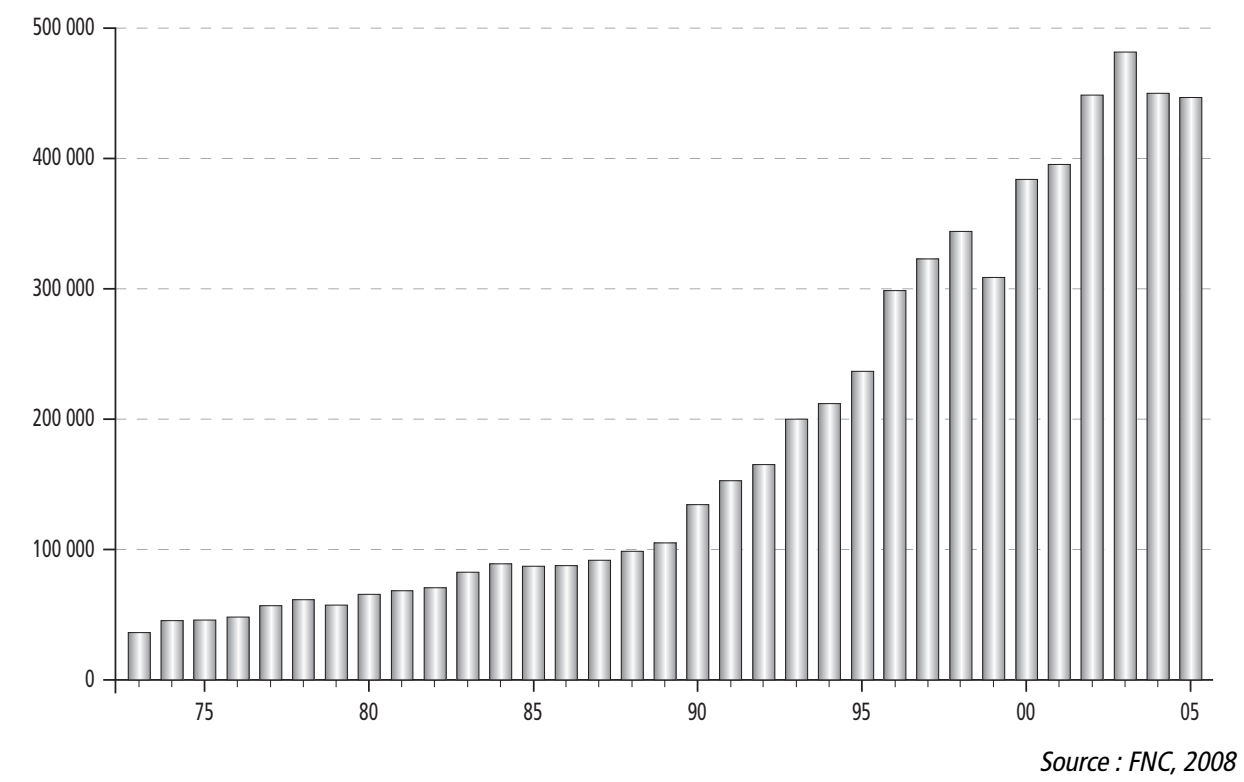

progressent. $10 \%$ de cerfs et $16 \%$ de chevreuils ont été abattus en plus. À l'exception des zones de montagne, il n'existe plus de prédateurs pour les cervidés. Seul l'homme peut donc gérer la dynamique des populations par ses prélèvements.

3. Le nombre des sangliers prélevés a fortement augmenté à partir de 1989 à 2003. Il est passé d'environ 100000 sangliers abattus en 1989 à presque 500000 en 2003. Entre 1993 et 2002, la progression a été de $11 \%$ (Pfaff et Saint Andrieux, 2008). À partir de 2003 cependant on observe une stabilisation.

Ces évolutions traduisent à la fois l'augmentation du nombre des grands gibiers en France et une intensification de l'activité de chasse. L'activité de chasse est plus intensive dans le sens où le nombre de grands gibiers abattus par chasseur s'en est trouvé inévitablement augmenté. Il y a moins de chasseurs, mais chaque chasseur abat plus de grands gibiers. Le nombre de prélèvements par chasseur a été en effet multiplié par 10 entre 1981 et 2005.

Ces évolutions du tableau de chasse s'expliquent par le statut du sanglier et l'existence ou non d'un plan de chasse (Pfaff et Andrieux, op. cit.). La hausse du niveau des prélèvements n'est donc pas indépendante de la loi. Pfaff et Saint Andrieux (Ibid.) constatent, tout d'abord, que le sanglier a été le plus chassé dans les départements qui l'ont classé comme espèce nuisible (tableau de chasse en augmentation). Ils observent aussi un effet des plans de chasse sur la dynamique des prélèvements. Le plan de chasse n'est obligatoire que pour les cervidés, car les règles législatives en matière de cervidés servent essentiellement à gérer la sous-population. Les règles en matière de sanglier sont faîtes, au contraire, pour gérer la surpopulation. En conséquence, l'augmentation conjointe du niveau des prélèvements et des indemnisations indique, cependant, que les populations de grands gibiers augmentent malgré les plans de chasse, les campagnes d'élimination pour le sanglier et une chasse plus intensive. 


\section{Évolution des indemnisations}

Ces explications ne portent, cependant, aucune attention aux effets des institutions sur les coûts d'opportunité des choix faits par les chasseurs et les agriculteurs. Elles font comme si la dynamique des dépenses d'indemnisation était indépendante des règles du jeu et de la structure des coûts et des bénéfices qu'elle impose (North, 1991). L'apport de la théorie économique à la compréhension de l'évolution du montant des indemnités versées par les chasseurs aux agriculteurs est de montrer l'effet du droit sur le coût d'opportunité d'un prélèvement et sur les coûts de coordination. Le droit influence les décisions en modifiant le système des incitations et de communication à partir duquel les agents évaluent les coûts d'opportunité de chacune de leurs actions et se coordonnent. La loi de 1844 a institué un permis de chasse. La loi de 1968 a dissocié le droit de prélèvement ou d'affût du droit de propriété sur la terre. Elle a aussi institué un modèle de responsabilité sociale. L'économiste cherche, dans ce cadre, à connaître les effets de telles règles sur le coût des prélèvements et in fine le nombre des grands gibiers et sur le montant et la répartition des coûts de précaution en présence d'un risque de dégâts de gibier entre les agriculteurs et les chasseurs.

\section{Coût d'un prélèvement, permis de chasse et dépenses d'indemnisation}

Le prix est la variable explicative clé de la théorie économique marginaliste et de son explication de la coordination de l'offre à la demande d'un bien. L'absence de prix est, en revanche, à l'origine de phénomène de discoordination. Sur un marché classique lorsque le prix augmente l'offre s'accroît. Si l'abattement du grand gibier était coordonné par un tel système de prix, à une augmentation de la demande d'abattement de la part de l'ensemble des personnes qui supportent les dommages liés aux dégâts occa- sionnés par le grand gibier correspondrait une hausse de l'offre des chasseurs qui ajusteraient ainsi leur activité à la demande. Le nombre des grands gibiers serait, dans ces conditions, régulé par le prix de l'abattement, autrement dit le consentement à payer des agents pour un tel service. Pour qu'un tel mécanisme s'applique, il faudrait, cependant, que les individus puissent librement négocier ce droit de prélèvement sur un marché. Cette liberté contractuelle est limitée par la loi sur la chasse de 1968 et l'avènement en 1844 du permis de chasse (Lois de décembre 1974 et mai 1975 pour sa forme actuelle).

Avant la loi de 1968, le droit reconnaissait à l'agriculteur un droit d'affût (Code rural, article 393, abrogé en 1968). « Tout propriétaire ou fermier pouvait repousser ou détruire, même avec des armes à feu, mais à l'exclusion du collet et de la fosse, les bêtes fauves qui portaient dommages à ses propriétés ». Il donnait une sorte de droit d'autodéfense aux agriculteurs qui pouvaient ainsi se protéger eux-mêmes de l'intrusion du grand gibier sur leur terre. Par ailleurs, il pouvait vendre ce droit à un chasseur ou acheter sa compétence. La loi de 1968 a rompu avec ce mode de régulation. Elle a dissocié le droit de propriété du droit d'affût. Elle a attribué aux chasseurs un droit de prélèvement et l'a retiré aux propriétaires fonciers, en abolissant le droit d'affût des agriculteurs ${ }^{7}$. Elle a retiré aux agriculteurs le droit de défendre la valeur de leur propriété contre les agressions commises par les grands gibiers.

7. Article L. 426-1 (Loi n ${ }^{\circ} 2005-157$ du 23 février 2005 art. 172 I Journal officiel du 24 février 2005). En cas de dégâts causés aux cultures ou aux récoltes agricoles soit par les sangliers, soit par les autres espèces de grand gibier provenant d'une réserve où ils font l'objet de reprise ou d'un fonds sur lequel a été exécuté un plan de chasse, l'exploitant qui a subi un dommage nécessitant une remise en état ou entrainant un préjudice de perte agricole peut en réclamer l'indemnisation à la fédération départementale ou interdépartementale des chasseurs. 
La loi de 1968 n'a pas abrogé le permis de chasse et a conduit les instances de régulation à instituer un bracelet ou timbre. Pour chasser le grand gibier, le chasseur doit acheter un permis de chasse, payer des bracelets sanglier, ou grands gibiers. Le coût du permis de chasser se compose d'une cotisation fédérale (environ 65 euros), d'une redevance cynégétique nationale (environ 194 euros) $)^{8}$, d'une redevance d'État (environ 9 euros), d'une cotisation nationale grand gibier (environ 45 euros) ${ }^{9}$ et selon les fédérations départementales un contrat d'assurance responsabilité civile chasse (environ 18 euros $)^{10}$. Le prix des timbres grands gibiers varie d'un département à l'autre et du type de permis de chasseur. Chaque département détermine sa politique, ce qui peut conduire certains d'entre eux à déterminer des stratégies tarifaires pour attirer des adhérents (et donc des cotisants) supplémentaires. Enfin, le département peut vendre des bracelets sangliers ou des bracelets grands gibiers. Instituer un timbre sanglier augmente ainsi le coût monétaire du prélèvement d'un sanglier. Il baisse dans le même temps le coût relatif du prélèvement du cerf ou du chevreuil.

L'ensemble de ces sommes ne constitue pas un prix de marché, car elle n'évolue pas en fonction de l'offre et de la demande de prélèvements, mais en fonction des besoins financiers des organismes de gestion de la chasse. L'établissement d'un permis de chasse instaure une contribution, une sorte de « prix » (en fait une expression monétaire tout au plus), mais celui-ci n'est pas assis sur un droit de propriété acquis sur le marché.

\footnotetext{
8. Cette redevance alimente le budget de l'Office national de la chasse et de la faune sauvage (ONFCS).

9. Cette cotisation participe au financement du service dégâts de la Fédération nationale des chasseurs (FNC). Cette cotisation nationale donne le droit de chasser le grand gibier dans n'importe quel département français.

10. Information de l'ONCFS, consultation de leur site en février 2009.
}

Il n'y a pas de marché des «droits de chasser », seulement un monopole sur la vente de permis de chasse dont disposent et gèrent les fédérations de chasseurs. En effet, des personnes de droit privé ne peuvent pas vendre leur «droit de chasser », car seules les fédérations de chasseurs disposent de l'autorisation de vendre des permis, assimilés à des droits (politiques et non assis sur une quelconque propriété $)^{11}$. Dans un système où le propriétaire foncier posséderait le droit de chasser, les choses seraient différentes. Ce dernier pourrait vendre ce droit, le commercialiser soit pour en tirer un profit, soit pour se protéger contre les dégâts de gibier. Un tel régime juridique rendrait possible la constitution d'un marché, car chaque propriétaire pourrait vendre ce droit en fonction de ces besoins (limiter les dégâts) et de la demande de droit de chasser des agents.

L'absence d'un prix de marché a pour conséquence de rendre impossible le calcul économique, parce qu'elle prive les agents de la connaissance sur leur disposition à payer pour prélever un grand gibier. Elle ne permet pas non plus de disposer d'informations sur les dispositions à payer des agriculteurs pour éviter les dégâts de gibier. On ne sait pas non plus combien les écologistes seraient prêts à payer pour éviter les prélèvements de gibier, pour acheter les droits de chasse (pour ne pas les utiliser). On ne sait pas, enfin, combien les chasseurs seraient prêts à recevoir pour renoncer à utiliser leur droit de chasse ou encore à le vendre à un autre chasseur qui proposerait un prix plus élevé.

Dans ces conditions, ce n'est pas l'absence de droits de propriété sur le grand gibier qui pose problème comme le soutient généralement la théorie des droits de propriété issue de la théorie de la tragédie des communs (Dowling, 1968), mais la limitation des droits d'usage de la propriété

11. On pourrait utiliser également la dénomination d'autorisation administrative. 
Figure 6. Le paradoxe du cadre juridique actuel : « augmenter la valeur des timbres diminue le niveau des prélèvements, augmente les dégâts de gibier et les dépenses d'indemnisation ”

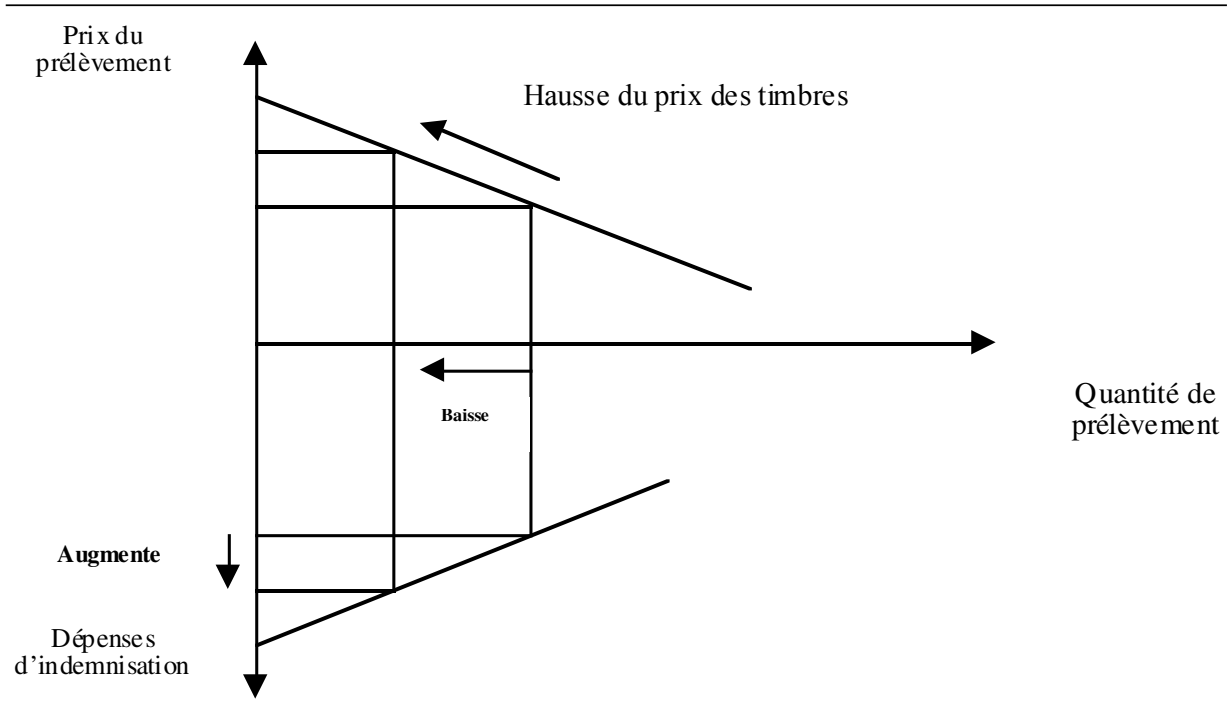

foncière. Le gibier peut rester un res nullius. Cependant, pour assurer une gestion économique du stock de gibier, un mécanisme de coordination s'avère nécessaire. En créant les conditions juridiques de l'émergence d'un profit par la vente des droits de chasse, la loi favoriserait l'activité productive des entrepreneurs et la constitution d'une chasse de marché (ou commerciale). La croissance des indemnisations traduit dans ces conditions l'effet d'une loi qui substitue au droit de chasse le permis de chasse.

L'établissement d'un permis de chasse et d'un système d'indemnisation financé par la vente d'un timbre ou d'un bracelet produit un effet sur le coût du prélèvement du grand gibier et in fine sur le nombre de contentieux relatifs au dégât de gibier.

L'institution d'un permis de chasse augmente le prix des prélèvements pour les propriétaires fonciers et les agriculteurs. Les agriculteurs ont perdu leur droit d'affût. Le permis de chasse oblige les individus à affecter plus de ressources pour se défendre. Il empêche aussi les agriculteurs ou les propriétaires de vendre leur droit d'affût à des chasseurs ou leur droit de chasse. Il bloque, en ce sens, le processus spontané de spécialisation qui caractérise les économies modernes.

L'institution d'un bracelet ou d'un timbre participe aussi à l'augmentation du prix du prélèvement. Il est, de surcroît, à l'origine d'un paradoxe. En effet, dans le système présent d'indemnisation, le prix des timbres et la vente des bracelets sangliers et grands gibiers financent les dépenses d'indemnisation. Leur évolution dépend principalement de celle du montant des indemnisations versées et liées aux dégâts de grand gibier et en particulier des sangliers. De la même manière, la variation du prix de la vignette fédérale répond à cette même exigence. Elle conduit à augmenter le prix du prélèvement du grand gibier pour les chasseurs, ce qui implique une diminution de la demande de prélèvements émanant des chasseurs. In fine, cela conduit à augmenter le nombre de grands gibiers et à favoriser la hausse des dégâts causés par le grand gibier et des dépenses d'indemnisation dans le moyen et long terme (figure 6).

La loi de 1968 crée, pour cette raison, un cercle vicieux du point de vue économique. L'absence de prix de marché oblige les 
RECHERCHES

Laurent CARNIS, François FACCHINI

Tableau 1. Règles de responsabilité en vigueur avant et après 1968

\begin{tabular}{|c|c|}
\hline Règles de responsabilité en vigueur avant 1968 & Règles de responsabilité en vigueur après 1968 \\
\hline Droit de chasser et responsabilité pour faute & Permis de chasse et responsabilité sociale \\
\hline Agriculteur - Propriétaire foncier & Agriculteur - Propriétaire foncier \\
\hline $\begin{array}{l}\text { - supporte le dommage sur son terrain } \\
\text { - est responsable des dommages sur le terrain des } \\
\text { autres } \\
\text { - intérêt à limiter le nombre des grands gibiers } \\
\text { pour limiter les risques de dégât (prélever, } \\
\text { clôturer, etc.) }\end{array}$ & $\begin{array}{l}\text { - droit à l'indemnisation en cas de dégâts de } \\
\text { grands gibiers }\end{array}$ \\
\hline Chasseur & Chasseur \\
\hline $\begin{array}{l}\text { - droit de chasse détenu par les propriétaires } \\
\text { fonciers } \\
\text { - peut offrir ses services aux propriétaires pour } \\
\text { abattre les grands gibiers nuisibles et/ou acheter } \\
\text { le droit d'affût aux propriétaires fonciers }\end{array}$ & $\begin{array}{l}\text { - doit acheter un permis de chasse pour prélever } \\
\text { du gibier } \\
\text { - doit collectivement indemniser les agriculteurs } \\
\text { en cas de dégâts de gibier (responsabilité } \\
\text { sociale) }\end{array}$ \\
\hline
\end{tabular}

autorités de la chasse à gérer de manière quantitative et comptable ce qui nuit à l'efficacité ultime de ses décisions. Le chasseur adapte son activité de chasse à cette information prix. Cependant, comme il ne s'agit pas d'un prix de marché fondé sur des droits de propriété bien définis, il prend une décision qui est contraire à son intérêt (qui conduit à augmenter les dépenses d'indemnisation et à accroître le prix de son loisir) et à celui des intérêts de la collectivité (augmentation des dégâts de gibier et ses coûts).

\section{Les effets d'un modèle de responsabilité sociale sur l'accroissement des indemnisations}

La loi de 1968 ne modifie pas seulement la relation qu'entretient le propriétaire foncier à son droit d'affût. Elle institue aussi une responsabilité sociale. Elle a fait passer d'un

Figure 7. Règle de responsabilité et dégâts de gibier

Niveau de précaution des agriculteurs

Niveau de précaution des chasseurs

Niveau d'activité des chasseurs

Niveau d'activité des agriculteurs

système de responsabilité pour faute à un système de responsabilité sociale (tableau 1). Elle responsabilise les chasseurs en leur faisant supporter les coûts d'une mauvaise gestion cynégétique (augmentation des dépenses d'indemnisation). Elle ne responsabilise pas, en revanche, le chasseur et les agriculteurs. Ceci constitue une explication possible de l'augmentation des dépenses d'indemnisation et du nombre des dégâts de gibier. Ces éléments d'explication doivent être mis en correspondance avec les évolutions du monde de la chasse.

\section{La théorie économique des règles de responsabilité et ses effets sur le calcul des agriculteurs}

La théorie économique de la responsabilité peut être mobilisée pour faciliter l'identification des effets associés aux règles de responsabilité sur les comportements des acteurs (Friedman, 2000 ; Cooter et Ulen, $2004)^{12}$. Cette théorie permet de prévoir un certain nombre de situations et d'évaluer ses effets sur les choix des acteurs. L'analyse

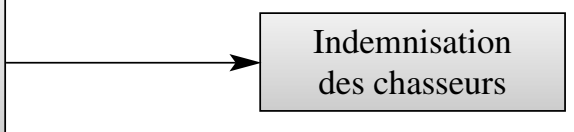

12. Pour éviter les lourdeurs de la démonstration, le lecteur est invité à se reporter à l'ouvrage de Shavell (1987) sur l'efficacité comparée des règles de responsabilité. On évoquera ici seulement les conclusions de cette approche. 
Tableau 2. L'hypothèse d'une règle de responsabilité limitée en matière d'indemnisation des dégâts de gibier

\begin{tabular}{c|c}
\hline Acteurs/ Règles & Responsabilité limitée \\
\hline Agriculteur & " est dédommagé s'il n'a pas fait de faute, \\
(victime) & autrement dit s'il s'est protégé de manière raisonnable » \\
\hline Chasseur & "doit payer s'il n'a pas pris les mesures de précaution raisonnable \\
pour éviter le dommage »
\end{tabular}

économique de la responsabilité fonde son approche sur les variables influençant le risque d'un dommage (ici le dégât causé par le gibier) et les coûts qui y sont associés. Le nombre de dégâts de gibier et leur importance peuvent être réduits par différents moyens (figure 7).

L'auteur du dommage (ici le chasseur) et son niveau d'activité sont évidemment concernés. L'importance du préjudice indemnisable peut aussi être contenue par un comportement approprié de la victime (l'agriculteur). Or la loi de 1968 considère comme auteur du dommage le groupe des chasseurs, et qu'il peut gérer par son activité de prélèvements les risques du dommage. La loi suppose qu'il peut gérer par son activité de prélèvements les risques de survenance du dommage. La loi n'envisage pas, cependant, les cas où l'activité et le comportement de la victime peuvent influer sur le niveau des risques. Certes, l'abattement (5\% des dommages) peut inciter l'agriculteur à maîtriser en partie les risques de dommage, mais cela reste très marginal. Au contraire, la loi de 1968 a procédé à un mouvement inverse en réduisant de manière substantielle le niveau de l'abattement, puisque ce taux était auparavant fixé à $20 \%$.

En conséquence, cette loi n'incite pas les agriculteurs à prendre des précautions puisqu'elle considère l'agriculteur comme n'étant jamais responsable des dommages subis (sauf si sa responsabilité est démontrée) ${ }^{13}$. En revanche, la loi a incité les chasseurs à prendre des mesures de précau-

13. Dans le cas d'une responsabilité démontrée de l'agriculteur, l'abattement peut être porté à $80 \%$. tion à travers des plans de chasse dont l'objectif est d'atteindre la quantité de grand gibier qui réduit le coût social. Elle les a obligés également à une meilleure organisation en définissant des schémas départementaux de gestion cynégétique, qui ont été créés par la loi chasse du 26 juillet $2000^{14}$. La loi de 1968 consiste donc à faire supporter la plus grande partie des coûts de précaution et des dommages sur les chasseurs.

Les coûts de précaution pour se protéger du grand gibier peuvent consister en la pose de clôtures, par la dissuasion olfactive et gustative (mise en place de substances répulsives), par la formation de petites parcelles de culture pour le gibier, le positionnement préférentiel de jachères adaptées à la faune sauvage à proximité des lieux fréquentés par les animaux, mais aussi par la plantation de cultures les moins attractives en bordure des forêts comme l'orge, le seigle ou les variétés de blé barbu. Toutes ces pratiques ont un coût qui est de fait supporté par les chasseurs. En somme, la loi de 1968 a non seulement redistribué la prise en charge financière des dommages de gibier vers les chasseurs, mais également conduit à déresponsabiliser les agriculteurs en les exonérant de prendre des mesures de précaution appropriées. En conséquence, si l'on admet que les précautions prises par les agriculteurs peuvent influencer les pertes directes liées aux dégâts de grand gibier, la règle de responsabilité actuelle est inefficace. Le niveau de précaution au niveau de la société

14. Guide méthodologique pour l'élaboration des schémas départementaux de gestion cynégétique (2005), disponible sur www.chasseurdefrance.com/ dossiers/frame.php?page $=5$. 
ne sera pas celui qui est économiquement optimal (Shavell, 1987).

La règle de responsabilité stricte en exonérant les agriculteurs conduit ces derniers à adopter un niveau d'activité trop important (Shavell, op. cit.). La règle de responsabilité la plus performante serait celle d'une responsabilité partagée obligeant chacune des parties à procéder à un minimum de dépenses de précaution, les obligeant ainsi à moduler leur activité productive respective. Il serait préférable, dans ces conditions, de mettre en place un principe de responsabilité limitée (tableau 2).

\section{Responsabilité sociale et comportements des chasseurs}

La loi de 1968 institue une sorte de responsabilité stricte, mais cette dernière est sociale ou collective. Elle ne rend pas le chasseur pris individuellement responsable des dégâts de gibier mais l'ensemble des chasseurs d'une fédération ou d'un groupe de chasseurs. Ce sont les chasseurs en tant que collectif, par l'entremise des fédérations départementales de chasse, et non un chasseur en particulier, qui sont responsables des dommages provoqués par le grand gibier. Les chasseurs paient à cet effet un timbre départemental. La loi de 1968 instaure ainsi la mutualisation obligatoire des risques entre les chasseurs. Mutualisation également par l'intermédiaire des permis de chasse nationaux, dont une partie des revenus est rétrocédée aux fédérations selon une procédure de calcul particulière pour pourvoir aux paiements des dégâts causés par le gibier. Mutualisation enfin par l'élaboration des plans de chasses départementaux qui par les revenus générés par les bracelets de certaines espèces permet d'indemniser les dommages causés par d'autres.

\section{La stratégie de passager clandestin}

La théorie économique a montré à partir de nombreux exemples que la socialisation des risques était à l'origine de comporte- ments « opportunistes ». La mutualisation des risques entre chasseurs peut conduire les chasseurs pris individuellement ou les associations communales de chasse à choisir une telle stratégie. La socialisation des risques favorise la prise de risque, car la partie (individu ou association communale de chasse) ne supporte pas l'intégralité des risques pris. L'agent sait aussi que si les autres prennent des risques inconsidérés, cela reviendra à lui en faire supporter une partie des coûts associés. L'agent a intérêt dans ces conditions à prendre également des risques, parce qu'il en tirera des bénéfices, mais n'en supportera pas toutes les conséquences. La loi de 1968 aura tendance à favoriser ce type de stratégie à l'intérieur du monde des chasseurs. On peut prendre le risque d'avoir une population de grands gibiers (ou de certaines espèces) trop importante sur son territoire de chasse, car les coûts d'une surpopulation seront gérés par l'ensemble des associations de chasse du département. Les bénéfices, en revanche, seront locaux. Le coût d'opportunité d'un prélèvement (temps passé) sera d'autant plus faible que la population de grand gibier est grande.

Une mauvaise gestion des risques de surpopulation n'est pas sanctionnée par la prise en charge directe de l'intégralité des dommages. On est en présence de la tragédie du « buffet à volonté ». Le chasseur paie une taxe d'un montant fixe qui ne correspond à aucune prestation particulière. Ce qu'il fait ensuite est indépendant de ce qu'il a payé. Le prix est forfaitaire. Il n'existe donc pas de relation entre le prix et l'activité de chasse. En conséquence, la loi de 1968 ne conduit pas les agents à atteindre le niveau de précaution permettant de limiter le coût de la gestion des dégâts de gibier parce qu'elle ne responsabilise pas le chasseur ou un groupe de chasseurs au niveau d'un territoire, mais les chasseurs en tant que collectif. Non seulement la loi de 1968 a transféré la prise en charge financière des 
dégâts de grand gibier vers un autre agent, le chasseur, mais en collectivisant le risque. La collectivisation du risque est sans aucun doute le prix à payer pour obtenir une mutualisation des risques pour faire face au paiement des indemnisations. Du point de vue de l'analyse économique, ce mode de régulation apparaît comme sous-optimal et ne constitue pas le meilleur moyen de réduire finalement l'importance des dégâts subis.

\section{Conclusion}

Cet article suggère donc que les mécanismes actuels d'indemnisation et les modalités de détermination de la responsabilité en matière de dégâts causés par le grand gibier constituent des éléments d'explication possibles de l'accroissement des dépenses d'indemnisation.

Il met en évidence les effets associés à l'absence de prix de marché, la séparation entre le droit de propriété foncière et le droit cynégétique, ainsi que l'augmentation du coût relatif de la chasse. La difficulté de procéder au calcul économique conduit à la production d'effets nuisibles (destruction de richesses et renchérissement de l'activité de chasse). La solution qui est esquissée consiste à réintégrer le droit cynégétique dans le droit foncier, pour qu'il ne devienne qu'un ou retrouve sa complétude. Cette réintégration implique donc d'attribuer la responsabilité des dommages au propriétaire de droit, qui sera alors enclin à arbitrer entre ses différentes formes d'intervention (indemniser les victimes des dommages voire les supporter directement, mettre en œuvre des stratégies de régulation en chassant ou en confiant cette activité à des chasseurs (professionnels ou amateurs, à des fédérations) avec lesquels il devra trouver des moyens de rémunération idoine.

Ces solutions découlent de l'analyse économique proposée. Il reste que le déclin de l'activité de chasse rend compte aussi des évolutions qui traversent une société. La chasse comme activité de loisirs est désormais en concurrence avec d'autres types de loisirs plus attractifs et plus individualisés (parc à thèmes, clubs de sport), moins contraignants (moins gourmands en temps, moindre exigence en termes de régularités), et moins coûteux socialement (la participation à certaines activités de chasse peut exiger une certaine cooptation de ses membres). En somme, l'activité de chasse implique donc d'autres types de coûts relatifs, que l'on pourrait qualifier de psychiques et qui ne sont pas directement monétaires. Ce renchérissement de l'activité de chasse se conjugue aux effets mentionnés précédemment.

Par ailleurs, l'activité de chasse s'insère dans un cadre organisationnel et institutionnel marqué, qui ne constitue pas le cœur de cette analyse, mais qui sans nul doute intervient dans la traduction du problème actuel. L'explication avancée ici n'épuise pas la complexité des enjeux et des problèmes, mais souligne la nécessité de prendre en considération la dimension économique de celui-ci pour y apporter des réponses adaptées.

Dans le cas où l'on se dirigerait vers une « chasse de marché », laquelle ne doit pas être confondue avec la chasse commerciale actuelle, les conditions d'implémentation d'un cadre juridique rénové soulèvent de nouveaux enjeux, notamment pour ce qui concerne les territoires caractérisés par des petites propriétés voir un morcellement du territoire. Se posera alors le problème, entre autres, de la coordination, auquel les mécanismes de prix pourront répondre, et aussi une difficulté dont la complexité ne doit pas être sous-estimée. Le nouveau régime de responsabilité devra établir les obligations pour le propriétaire, lorsque celui-ci ne désirera pas réguler le grand gibier sur sa propriété ou lorsqu'il aura fait preuve d'une négligence.

Enfin, concernant ce changement juridique sur les activités de chasse et son organisation actuelle de nouvelles réflexions 
doivent être menées. Celles-ci seront-elles amenées à évoluer et à s'adapter ? Si l'activité de chasse continue à tendre vers un moindre attrait pour les nouvelles générations, l'émergence d'une nouvelle catégorie de « chasseurs professionnels » deviendrat-elle inéluctable et comment s'accordera-telle avec ce cadre juridique ? Comment sera-t-elle alors financée ? Ces nouvelles questions ne constituent pas la problématique de cette étude, mais l'analyse qui est défendue les met d'autant plus en exergue et pourrait justifier l'entreprise de nouvelles investigations plus approfondies.

Les auteurs tiennent à remercier B. Guibert pour sa disponibilité et les informations transmises qui ont contribué à la réalisation de cet article. Les auteurs restent les seuls responsables des arguments avancés ici, lesquels ne sauraient engager leur organisation de rattachement respective.

\section{RÉFÉRENCES BIBLIOGRAPHIQUES}

Baubet E., Vassant J., Brandt S., Maillard D. (2008). Connaissances sur la biologie du sanglier : utilisation de l'espace et régime alimentaire. Colloque modalités de gestion du sanglier, Fédération Nationale des Chasseurs - Office National de la Chasse et de la Faune Sauvage, CEDEROM, p. 56-59.

Bedarida G., François A. (2008). Impact de la baisse du nombre de chasseurs sur la gestion du sanglier et perspectives. Colloque modalités de gestion du sanglier, Fédération Nationale des Chasseurs - Office National de la Chasse et de la Faune Sauvage, CEDEROM, p. 206214.

Le Goffe P., Ropars C. (2008). Analyse économique du bilan chiffré des dégâts agricoles. Colloque modalités de gestion du sanglier, Fédération Nationale des Chasseurs - Office National de la Chasse et de la Faune Sauvage, CEDEROM, p. 79-86.

Chassin N. (2001). Aspects juridiques de la préservation des grands prédateurs en France : les cas du loup et de l'ours. Mémoire de DEA de droit public sous la direction de Gaïa P., Université d'AixMarseille III.

Charlez A. (1994). Dommages causés par les espèces sauvages et indemnisation. Bulletin mensuel de l'Office National de la Chasse, $\mathrm{n}^{\circ} 192$, p. 67-69.
Cooter R., Ulen T. (2004). Law and Economics. Pearson International, $4^{\text {th }}$ edition. Delfour O. (1998). La conservation des espèces menacées d'extinction. Étude de droit comparé : Espagne, États-Unis, France, Grande-Bretagne, Suisse. Thèse de doctorat, Université Paris 1 Panthéon - Sorbonne.

Desbois D., Legris B. (édition 2007). Prix et coûts de production de six grandes cultures : blé, maïs, colza, tournesol et pomme de terre. L'agriculture, nouveaux défis, disponible en ligne.

Dowling J. (1968). Individual Ownership and The Sharing of Game in Hunting Societies. American Anthropologist, vol. 70, $\mathrm{n}^{\circ} 3$, p. 502-507.

Friedman D. (2000). Law's Order, What Economics Has to Do with Law and Whys It Matters. Princeton University Press. Guibert B. (2008). Bilan de l'indemnisation des dégâts agricoles de sangliers. Colloque modalités de gestion du sanglier, Fédération Nationale des Chasseurs - Office National de la Chasse et de la Faune Sauvage, CEDEROM, p. 7378.

Malafosse (de) J. (1989). Nature et liberté, les acquis de la révolution française. Revue Droit Rural, ${ }^{\circ} 178$, p. $486-494$. North D.-C. (1991). Institutions. Journal of Economics Perspectives, vol. 5, $\mathrm{n}^{\circ} 1$, p. 97-112. 
Pfaff E., Saint Andrieux C. (2008). Le développement du sanglier en France. Colloque sur les modalités de gestion du sanglier, Fédération Nationale des Chasseurs-Office National de la Chasse et de la Faune Sauvage, CEDROM, p. 3-11. Vassant J., Brandt S. (2008). Modalités de prévention des dégâts de sangliers aux cultures agricoles. Colloque modalités de gestion du sanglier, Fédération Nationale des Chasseurs - Office National de la Chasse et de la Faune Sauvage, CEDEROM, p. 100-105.

Shavell S. (1987). Economic Analysis of Accident Law. Harvard University Press. 